\title{
Atributos de qualidade, compostos bioativos e atividade antioxidante de frutos de uvaieira durante a maturação
}

\section{Attributes of quality, bioactive compounds and antioxidant activity of uvaia fruit during maturation}

\author{
José Roberto Chaves Neto \\ Universidade Federal de Santa Maria (UFSM) \\ E-mail: jose.chavesneto@gmail.com \\ OrclD: https://orcid.org/0000-0003-1799-4630
}

Silvanda de Melo Silva

Universidade Federal da Paraíba (UFPB)

E-mail: silvandasilva@gmail.com

OrclD: https://orcid.org/0000-0003-2106-6458

Renato Lima Dantas

Faculdade Nova Esperança, João Pessoa, PB

E-mail: renatodsobrinho@gmail.com

OrcID: https://orcid.org/0000-0002-5464-9476

Resumo: Este trabalho teve como objetivo avaliar as mudanças nos aspectos de qualidade, compostos bioativos e atividade antioxidante de frutos de uvaieira (Eugenia pyriformis Cambess) em diferentes estádios de maturação. Os frutos foram avaliados quanto à massa fresca, comprimento e diâmetro, rendimento de polpa, sólidos solúveis (SS), acidez titulável (TA), relação SS/TA, ácido ascórbico, carotenoides totais, flavonoides amarelos e atividade antioxidante (DPPH). O delineamento experimental foi o inteiramente casualizado. Os frutos da uvaieira apresentam aumento na massa fresca e rendimento de polpa durante a maturação, atingindo médias de $11,69 \mathrm{~g}$ e $65,49 \%$ ao final, respectivamente. A uvaia é um fruto de elevada acidez, a qual diminui com a maturação, paralelamente ao aumento nos sólidos solúveis. A capacidade antioxidante total aumenta com o avanço da maturação da uvaia, em paralelo ao aumento dos teores de carotenoides e flavonoides, caracterizando as mudanças mais marcantes dos estádios finais da maturação.

Palavras-chave: Eugenia pyriformis Cambess, carotenoides, flavonoides, capacidade antioxidante, DPPH

\begin{abstract}
The objective of this work was to evaluate the changes in the aspects of quality, bioactive compounds and antioxidant activity of 'uvaia' fruits (Eugenia pyriformis Cambess) in different stages of maturation. Fruits were evaluated with regard to the fresh mass, length and diameter, pulp yield, soluble solids (SS), titratable acidity (TA), SS/TA ratio, ascorbic acid, total carotenoids, yellow flavonoids and antioxidant activity (DPPH). The experimental design was completely randomized. 'Uvaia' fruits presented increases in fresh mass and pulp yield during maturation with mean values of $11.69 \mathrm{~g}$ and $65.49 \%$, respectively he end of maturation. The 'uvaia' is a fruit of high acidity, which decreases with maturation, in parallel to the increase in soluble solids content. The total antioxidant activity increases with the advance of maturation, in parallel with the increase of carotenoid and flavonoid contents, characterizing the most striking changes in the final stages of maturation.
\end{abstract}

Keywords: Eugenia pyriformis Cambess, carotenoids, flavonoids, antioxidant activity, DPPH. 
Introdução

A uvaia (Eugenia pyriformis Cambess) é uma espécie frutífera pertencente ao gênero Eugenia, família das Mirtáceas, também conhecida popularmente como uvalha, uvaia-do-mato, ou ubaia, orvalha, uvalha-do-campo, uvaieira, uvalheira entre outros, o nome deriva de iwa'ya da língua tupi e significa 'fruto ácido'. A uvaieira é uma planta aromática de porte arbóreo, que pode medir de 5 a 15 m de altura, nativa do Brasil, encontrada principalmente na região da Mata Atlântica, desde regiões litorâneas sul do país até o norte, apresentando frutificação precoce e abundante, que se desenvolve muito bem nas condições de clima e da região Nordeste. Esta espécie é amplamente utilizada em programas de reflorestamento e em áreas metropolitanas (Andersen \& Andersen, 1989; Mattos, 1988; Landrum \& Kawasaki, 1997; Lorenzi, 2000).

O fruto da uvaieira é classificado como do tipo baga, de casca pilosa e coloração amarela ou alaranjada, de formato piriforme, redondo ou oval, de polpa aquosa e sabor ácido levemente adocicado, devido ao balanço entre os ácidos orgânicos e os sólidos solúveis. A polpa fresca de uvaia apresenta pH médio de variando de 2,57 a 3,15, acidez titulável de 3,98 \% de ácido cítrico e teor de sólidos solúveis de $9^{\circ}$ Brix (Scalon et al., 2004; Silva et al., 2018a; Zilo et al., 2013). Diversos autores mostram que as características de qualidade da uvaia variam de acordo com alguns fatores como região de origem, maturação dos frutos, sendo afetada pela forma de processamento (Silva et al., 2018a; Silva et al., 2018b). Estes frutos possuem sabor e aroma agradável, podem ser consumidos frescos ou processados na forma de sucos, licores, cervejas, vinagres, sorvetes, iogurtes, geléias e doce em pasta, sendo também usada na medicina popular no tratamento de gripe, febre e disenteria. (Andersen \& Andersen, 1989; Sobral et al., 2015).

Durante o desenvolvimento dos frutos ocorrem diferentes estágios fisiológicos, a partir da divisão, seguido do alongamento celular, que precedem as fases de pré-maturação, maturação, amadurecimento, culminando com a senescência (Wills \& Golding, 2016). As características físicas, químicas e sensoriais que tornam o fruto atrativo para o consumo, são desenvolvidas no processo do amadurecimento, que é marcado por eventos bioquímicos e fisiológicos simultâneos geneticamente controlados que faz parte do desenvolvimento do fruto. Poucos estudos têm sido realizados acerca dos aspectos de desenvolvimento, maturação e amadurecimento de uvaia (Jacomino et al., 2018).

De acordo com Scalon et al. (2004), a qualidade de um determinado fruto está relacionada ao seu tamanho, forma e coloração além das características físicas e composição química da polpa, oferecendo aos frutos e aos seus derivados a qualidade sensorial e nutricional, responsável pela sua aceitação definitiva no mercado. O estádio de maturação em que um fruto é colhido determina o perfil de compostos presentes na polpa deste, afetando a quantidade e o papel que desempenham na qualidade dos frutos após retirados da planta (Wills \& Golding, 2016).

As frutas são consideradas fontes ricas de compostos com potencial atividade funcional, como o ácido ascórbico, os ácidos fenólicos e os seus derivados que apresentam ação benéfica à saúde (Sellappan et al., 2002; Ali et al., 2008; Lu et al., 2011). Tem sido reportado que a polpa dos frutos de uvaieira apresenta um perfil de compostos bioativos, tais como carotenoides, flavonoides, compostos fenólicos e taninos, além de minerais como ferro e cálcio (Karwowski et al., 2013; Silva et al., 2018a).

Diversas pesquisas mostram que a uvaia possui elevado poder antioxidante em comparação com outras frutas brasileiras, bem como atividade anti-inflamatória (Haminiuk et al., 2011; Pereira et al., 2012; Rufino et al., 2010). A uvaia é um fruto que possui capacidade antioxidante benéfica, devido ao conteúdo de compostos bioativos presentes em sua polpa, o principal composto com esta capacidade, é o ácido ascórbico que pode variar entre 39,3 a 100,7 mg $100 \mathrm{~g}^{-1}$ de massa fresca. Porém também apresentam níveis elevados de compostos fenólicos que oscila entre 27,5 a $127 \mathrm{mg}$ $100 \mathrm{~g}^{-1}$ GAE massa fresca, e carotenoides com 909,33 e 1306,6 $\mu \mathrm{g} 100 \mathrm{~g}^{-1}$ de massa fresca (Rufino et al., 2010; Pereira et al., 2012; Silva et al., 2014; Zillo et al., 2014). Seifried et al. (2007) relatam

Agrarian, Dourados, v. 13, n. 49, p. 296-308, 2020. 
que estas substâncias apresentam a capacidade de reduzir o risco do desenvolvimento de doenças cardiovasculares, cânceres e outras doenças neurodegenerativas, devido sua ação antioxidante.

Entretanto, os frutos da uvaieira apresentam curta vida útil quando armazenados sob condições de temperatura ambiente, requerendo a aplicação de métodos de conservação póscolheita (Silva et al., 2018b). Assim, a determinação do ponto de colheita é estratégia chave para que se tenha maior durabilidade com qualidade após a colheita. Neste contexto, objetivou-se avaliar as mudanças nas características físicas, físico-químicas e na capacidade antioxidante durante a maturação de frutos de uvaieira (Eugenia pyriformis Cambess).

\section{Material e Métodos \\ Seleção e análise dos frutos}

Os frutos foram colhidos de plantas de ocorrência espontânea no município de Cuité de Mamanguape, PB, no horário da manhã e levados para o Laboratório de Biologia e Tecnologia de Pós Colheita (LBTPC), da Universidade Federal da Paraíba, onde foram lavados e selecionados, considerando a ausência de danos físicos. Em seguida, foram selecionados quanto ao estádio de maturação com base na coloração da casca e agrupados nos estádios: Verde (V), Início de Pigmentação Amarela (IP), Predominante Amarela (PA) e Totalmente Amarela (TA).

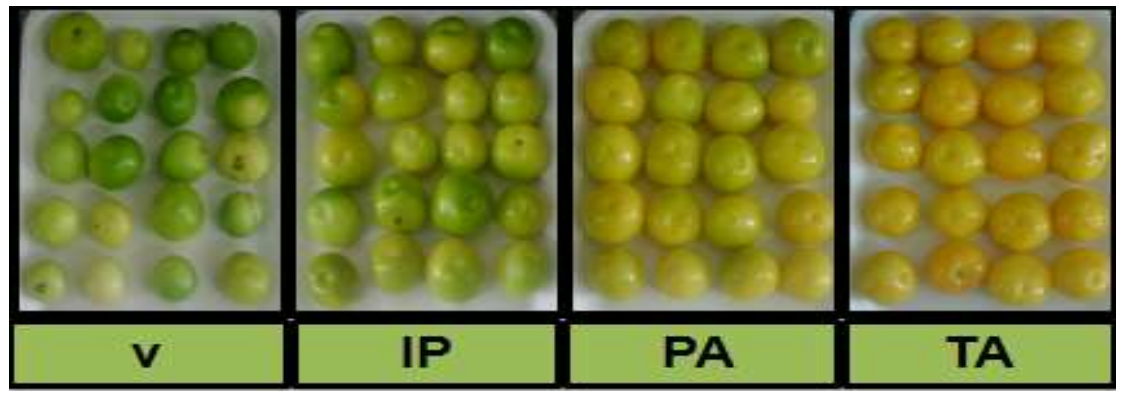

Figura 1. Evolução da maturação de frutos de uvaieira (Eugenia pyriformis Cambess). V (verde), IP (início de pigmentação amarela), PA (predominantemente amarelo) e TA (totalmente amarela).

\section{Análises}

\section{Atributos físicos}

Para as avaliações físicas foram utilizados 20 frutos, sendo considerado cada fruto uma repetição. |Estes foram avaliados quanto: comprimento e o diâmetro, com auxílio de um paquímetro digital (Within $300 \mathrm{~mm}$ ), e os resultados expressos em milímetros $(\mathrm{mm})$; massa fresca com balança semi-analítica (A42207c - Bel Engeneering), e os resultados expressos em gramas (g); firmeza de frutos íntegros através de um Penetrômetro de bancada digital, Magness Taylor Pressure Tester, região de inserção de 2/16 polegadas de diâmetro, duas leituras por fruto e os resultados expressos em Newton (N). O rendimento de polpa foi determinado através do seu isolamento e subsequente pesagem em balança semi-analítica (A 42207c - Bel Engeneering), e os resultados expresso em porcentagem (\%).

\section{Atributos físico-químicos}

Os frutos foram processados e a polpa homogeneizada para compor triplicata de cerca de $250 \mathrm{~g}$ para cada estádio de maturação. O potencial hidrogeniônico $(\mathrm{pH})$ foi determinado por meio

Agrarian, Dourados, v. 13, n. 49, p. 296-308, 2020. 
de um potenciômetro digital (Hanna, Singapura), segundo AOAC (2016). A acidez titulável (AT) foi determinada por titulometria com $\mathrm{NaOH} \mathrm{0,1} \mathrm{M,} \mathrm{de} \mathrm{acordo} \mathrm{com} \mathrm{Brasil} \mathrm{(2008),} \mathrm{resultados} \mathrm{expressos}$ em \% de ácido cítrico. Sólidos solúveis (SS), determinados com o auxílio de um refratômetro digital (Krüss-Optronic, Hamburgo, Alemanha), conforme Brasil (2008), expresso em \%. Já a relação SS/AT (ratio) foi determinada pela relação entre os SS e AT.

\section{Compostos bioativos e atividade antioxidante}

Para a quantificação do ácido ascórbico, homogeneizou-se $1 \mathrm{~g}$ de amostra em $50 \mathrm{~mL}$ de solução de ácido oxálico $0,5 \%$ e titulada com a solução DFI (2,6 dicloro-fenol-indofenol 0,002 \%), até a obtenção da coloração rósea claro permanente, sendo o ponto de viragem detectado visualmente, conforme Strohecker e Henning (1967), sendo os resultados expressos em mg.100 g ${ }^{1}$ de polpa.

Os conteúdos de carotenoides totais, foi determinado por espectrofotometria a $450 \mathrm{~nm}$, utilizando-se solução extratora de hexano 98,5\% (PA), conforme Higby (1962). Os flavonoides amarelos (mg.100 g ${ }^{-1}$ ), foi determinado por espectrofotometria a $374 \mathrm{~nm}$, utilizando-se solução extratora de etanol PA + $\mathrm{HCl} 1 \mathrm{M}(85: 15)$, seguindo a metodologia de Francis (1982), sendo os resultados expressos em $\mu \mathrm{g} .100 \mathrm{~g}^{-1}$ de polpa.

A capacidade antioxidante total da polpa foi determinada estimando-se o valor de EC50 (g de polpa.g de $\left.\mathrm{DPPH}^{-1}\right)$ através do método de sequestro do radical livre DPPH $\left(1,1^{\prime}\right.$-diphenil-2picrilhidrazil), conforme metodologia utilizada por Rufino et al. (2009).

\section{Delineamento experimental e análise estatística}

O delineamento utilizado foi o inteiramente casualizado e os resultados foram submetidos à análise de variância (ANOVA) e as médias comparadas pelo teste de Tukey a $5 \%$ de probabilidade de erro $(p \leq 0,05)$. Adicionalmente, os dados foram submetidos à análise multivariada, onde o agrupamento hierárquico foi conduzido com o JMP® 10.0.0 (SAS Institute Inc. 2012), utilizando agrupamento two-way para variáveis dependentes e independentes pelo método de Ward e considerando o critério cúbico de agrupamento.

\section{Resultados}

\section{Atributos físicos}

Foram observadas diferenças $(p \leq 0,05)$ entre os estádios de maturação avaliados, para as características comprimento, diâmetro, massa fresca, firmeza e rendimento de polpa. A precisão experimental avaliada pelo coeficiente de variação (CV) variou entre as características. O maior valor de CV foi observado para a massa fresca do fruto $(19,24 \%)$ e o menor, para o diâmetro $(8,34 \%)$, não ocorrendo nenhuma interferência que comprometesse o prosseguimento do ensaio (Tabela 1).

O comprimento, o diâmetro, a massa fresca e o rendimento de polpa dos frutos da uvaieira aumentaram, com exceção da firmeza, que reduziu com o avanço da maturação dos frutos de uvaieira (Tabela 1).

A amplitude para o comprimento e diâmetro dos frutos variou de 23,37 a 27,06 mm e 24,18 a $29,90 \mathrm{~mm}$ com média geral de 25,96 e $27,61 \mathrm{~mm}$, respectivamente (Tabela 1). Os frutos nos estádios de maturação IP, PA e TA destacaram-se por terem os maiores comprimentos, enquanto os dos estádios PA e TA foram os de maiores diâmetros $(28,19$ e 29,90 mm), respectivamente, sendo iguais entre si e diferindo dos demais estádios.

A massa fresca aumentou com o avanço da maturação, onde o estádio TA apresentou à maior média de 14,56 g, sendo a menor observada no estádio $\mathrm{V}$, com 8,36 g. Para o rendimento de

Agrarian, Dourados, v. 13, n. 49, p. 296-308, 2020. 
polpa (\%) observou-se que ocorreu aumento com o avanço da maturação, com valores variando de 59,02 a 75,69 \% (V e TA) e média de 65,69 \% (Tabela 1).

A firmeza dos frutos de uvaieira diminuiu ao longo da maturação, sendo que os valores reduziram progressivamente de $125,60 \mathrm{~N}$ em frutos do estádio $\mathrm{V}$ para $10,29 \mathrm{~N}$, no estádio TA (Tabela 1).

Tabela 1. Mudanças nas características físicas durante a maturação de frutos da uvaieira (Eugenia pyriformis Cambess)

\begin{tabular}{cccccc}
\hline $\begin{array}{c}\text { Estádio de } \\
\text { Maturação }\end{array}$ & $\begin{array}{c}\text { Comprimento } \\
(\mathbf{m m})\end{array}$ & $\begin{array}{c}\text { Diâmetro } \\
(\mathbf{m m})\end{array}$ & $\begin{array}{c}\text { Massa } \\
\text { fresca } \mathbf{( g )}\end{array}$ & $\begin{array}{c}\text { Rendimento } \\
\text { de polpa }(\%)\end{array}$ & $\begin{array}{c}\text { Firmeza } \\
(\mathbf{N})\end{array}$ \\
\hline V & $23,37 \mathrm{~B}$ & $24,18 \mathrm{C}$ & $8,37 \mathrm{C}$ & $59,02 \mathrm{~B}$ & $125,60 \mathrm{~A}$ \\
IP & $25,71 \mathrm{~A}$ & $27,58 \mathrm{~B}$ & $10,95 \mathrm{~B}$ & $50,78 \mathrm{C}$ & $48,95 \mathrm{~B}$ \\
$\mathrm{PA}$ & $27,24 \mathrm{~A}$ & $28,19 \mathrm{AB}$ & $12,18 \mathrm{~B}$ & $72,96 \mathrm{~A}$ & $12,42 \mathrm{C}$ \\
TA & $27,06 \mathrm{~A}$ & $29,90 \mathrm{~A}$ & $14,57 \mathrm{~A}$ & $75,69 \mathrm{~A}$ & $10,29 \mathrm{C}$ \\
\hline Média Geral & 25,96 & 27,61 & 11,69 & 65,49 & 45,87 \\
CV (\%) & 10,89 & 8,34 & 19,24 & 12,31 & 15,35 \\
\hline
\end{tabular}

V-Verde; IP - início de Pigmentação Amarela; PA - Predominante Amarela e TA - Totalmente Amarela. *Médias seguidas de mesma letra maiúscula na coluna não diferem entre si pelo teste de tukey ao nível de $5 \%$ de probabilidade de erro.

\section{Atributos físico-químicos}

Constatou-se que houve diferença $(p \leq 0,05)$ entre os estádios de maturação avaliados, para as características $\mathrm{pH}$, acidez titúlavel (AT) e relação sólidos solúveis/ acidez titúlavel (SS/AT). A precisão experimental, avaliada pelo coeficiente de variação (CV), variou entre as características, sendo que o maior valor de CV foi observado para a relação sólidos solúveis/ acidez titúlavel $(11,70$ $\%)$ e o menor, para o $\mathrm{pH}(2,83 \%)$, não sendo constatado nenhuma interferência que comprometesse o prosseguimento do ensaio (Tabela 2).

Quanto a avaliação da qualidade interna dos frutos de uvaiaeira avaliados neste trabalho verificou-se que o $\mathrm{pH}$, os SS e a relação SS/AT aumentaram e a AT reduziu ao longo da maturação dos frutos de uvaieira

(Tabela 2). $\mathrm{O}$ pH da polpa dos frutos de uvaieira partiu de 3,01 para 3,30, do estádio $\mathrm{V}$ para o TA, respectivamente. Já para o conteúdo de SS, observou-se teores médios variando de 7,90\% no estádio $\vee$ a 9,00 \% no estádio TA, apresentando média geral de $8,34 \%$, porém não diferindo entre os estádios de maturação.

A acidez titúlavel variou entre os estádios de maturação avaliados, observando uma sutil redução com o avanço da maturação, onde o estádio $V$ foi superior aos demais, apresentando a maior média (3,44 g.100 g-1 ácido cítrico). A relação SS/AT dos frutos de uvaieira variou entre o mínimo de 2,30 e o máximo de 3,34 , diferindo $(p \leq 0,05)$ entre os estádios de maturação avaliados, sendo as maiores médias para o IP, PA e TA, não sendo observada diferenças entre estes $(p \leq 0,05)$ respectivamente (Tabela 2 ). 
Tabela 2. Mudanças nas características físico-químicas durante a maturação de frutos da uvaieira (Eugenia pyriformis Cambess)

\begin{tabular}{ccccc}
\hline Estádio de Maturação & $\mathbf{p H}$ & $\mathbf{S S}(\%)$ & $\begin{array}{c}\text { Acidez Titulável } \\
\mathbf{( g . 1 0 0} \mathbf{g}^{-1} \text { ác. cítrico) }\end{array}$ & SS/AT \\
\hline V & $3,01 \mathrm{~B}$ & $7,90 \mathrm{~A}$ & $3,44 \mathrm{~A}$ & $2,30 \mathrm{~B}$ \\
IP & $3,05 \mathrm{~B}$ & $8,33 \mathrm{~A}$ & $2,58 \mathrm{~B}$ & $3,26 \mathrm{~A}$ \\
PA & $3,14 \mathrm{AB}$ & $8,13 \mathrm{~A}$ & $2,71 \mathrm{~B}$ & $3,02 \mathrm{AB}$ \\
TA & $3,30 \mathrm{~A}$ & $9,00 \mathrm{~A}$ & $2,71 \mathrm{~B}$ & $3,34 \mathrm{~A}$ \\
\hline Média Geral & 3,12 & 8,34 & 2,86 & 2,98 \\
CV (\%) & 2,83 & 5,95 & 6,26 & 11,70 \\
\hline
\end{tabular}

V - Verde; IP - início de Pigmentação Amarela; PA - Predominante Amarela e TA - Totalmente Amarela. *Médias seguidas de mesma letra maiúscula na coluna não diferem entre si pelo teste de tukey ao nível de $5 \%$ de probabilidade de erro.

Tabela 3. Mudanças nos conteúdos médios de ácido ascórbico, carotenoides totais, flavonoides amarelos e na capacidade antioxidante total (AAT) durante a maturação de frutos da uvaieira (Eugenia pyriformis Cambess)

\begin{tabular}{|c|c|c|c|c|}
\hline $\begin{array}{l}\text { Estádio de } \\
\text { Maturação }\end{array}$ & $\begin{array}{c}\text { Ácido } \\
\text { Ascórbico } \\
\left(\mathrm{mg} 100 \mathrm{~g}^{-1}\right)\end{array}$ & $\begin{array}{l}\text { Carotenoides } \\
\text { totais }\left(\mu \mathrm{g} 100 \mathrm{~g}^{-1}\right)\end{array}$ & $\begin{array}{l}\text { Flavonoides amarelos } \\
\qquad\left(\mathrm{mg} \mathrm{100g}^{-1}\right)\end{array}$ & $\begin{array}{c}\text { Capacidade } \\
\text { Antioxidante } \\
\text { EC50 (g de polpa.g } \\
\text { de } \text { DPPH }^{-1} \text { ) }\end{array}$ \\
\hline $\mathrm{V}$ & $3,81 \mathrm{~A}$ & $4,46 \mathrm{~B}$ & $0,89 B$ & $6417,36 \mathrm{~A}$ \\
\hline IP & $4,78 \mathrm{~A}$ & $7,81 \mathrm{~B}$ & $0,96 \mathrm{~B}$ & $6168,27 A$ \\
\hline PA & $7,01 \mathrm{~A}$ & $14,57 \mathrm{AB}$ & $1,69 \mathrm{~A}$ & $4684,36 \mathrm{~B}$ \\
\hline TA & $8,51 \mathrm{~A}$ & $24,81 \mathrm{~A}$ & $1,02 \mathrm{~B}$ & $3837,83 \mathrm{C}$ \\
\hline Média Geral & 6,03 & 12,91 & 1,14 & 5276,95 \\
\hline CV (\%) & 6,41 & 13,94 & 14,64 & 5,45 \\
\hline
\end{tabular}

V - Verde; IP - início de Pigmentação Amarela; PA - Predominante Amarela e TA - Totalmente Amarela. *Médias seguidas de mesma letra maiúscula na coluna não diferem entre si pelo teste de tukey ao nível de $5 \%$ de probabilidade de erro.

\section{Compostos bioativos e Atividade Antioxidante}

Com o avanço da maturação, constatou-se que houve aumento no conteúdo de ácido ascórbico, carotenoides totais, flavonoides amarelos e capacidade antioxidante total (AAT), não sendo observado diferença $(p \leq 0,05)$ entre os estádios de maturação apenas para o conteúdo ácido ascórbico (Tabela 3).

O conteúdo de ácido ascórbico de frutos de uvaieira variou de 3,81 a $8,51 \mathrm{mg} .100 \mathrm{~g}^{-1}$ de ácido ascórbico, com média geral de $6,03 \mathrm{mg} 100 \mathrm{~g}^{-1}$ de ácido ascórbico, porém não diferiu entre os estádios de maturação (Tabela 3).

Por sua vez, o conteúdo de carotenoides totais aumentou com o avanço da maturação, partindo de 4,46 para $24,81 \mu \mathrm{g} .100 \mathrm{~g}^{-1}$, do estádio $\mathrm{V}$ para o TA, respectivamente, seguido de redução para $14,57 \mu \mathrm{g} \cdot 100 \mathrm{~g}^{-1} \mathrm{no}$ estádio TA. (Tabela 3 ).

O conteúdo de flavonoides amarelos apresentou o mesmo comportamento que os carotenoides, visto que ocorreu acréscimo com o avanço da maturação, partindo de 0,89 para $1,69 \mathrm{mg} \cdot 100 \mathrm{~g}^{-1}$ do estádio $\mathrm{V}$ para

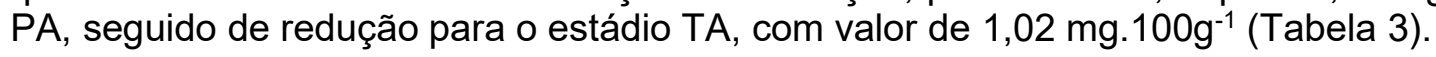

Quanto à AAT da polpa de frutos de uvaieira observou-se notadamente redução na quantidade de polpa necessária para reduzir em $50 \%$ o radical livre DPPH com o avanço da maturação (Tabela 3). Constatou-se aumento na AAT com o avanço da maturação, onde a polpa dos frutos do estádio TA apresentaram a maior

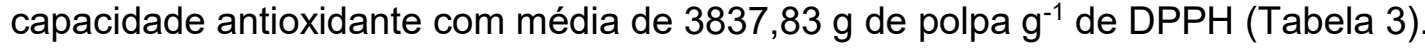

\section{Análise multivariada}

Com base na análise multivariada através do mapa de cores, onde foi agrupado para os diferentes estádios de maturação os atributos físicos, físico-químicos, compostos bioativos e capacidade antioxidante, constatou-se que a contribuição dos atributos físicos e físico-químicos da uvaia foi significativa para distinguir a qualidade dos frutos durante a maturação, exceto para a firmeza e a acidez titulável da polpa dos frutos que foi mais intensa nos frutos imaturos (estádio V). 
Para as características físicas comprimento, diâmetro, massa fresca e rendimento a maior intensidade foi observada entre os estádios mais avançados de maturação (estádio PA e TA) (Figura 2).

Os conteúdos de ácido ascórbico, carotenoides totais, flavonoides amarelos e capacidade antioxidante total (DPPH) tiveram a maior intensidade evidenciada próximo a maturação plena (estádio PA e TA). Notou-se que a capacidade antioxidante total em uvaia pode serexpressada principalmente pelos conteúdos de flavonoides amarelos, carotenoides totais e ácido ascórbico e da polpa (Figura 2).

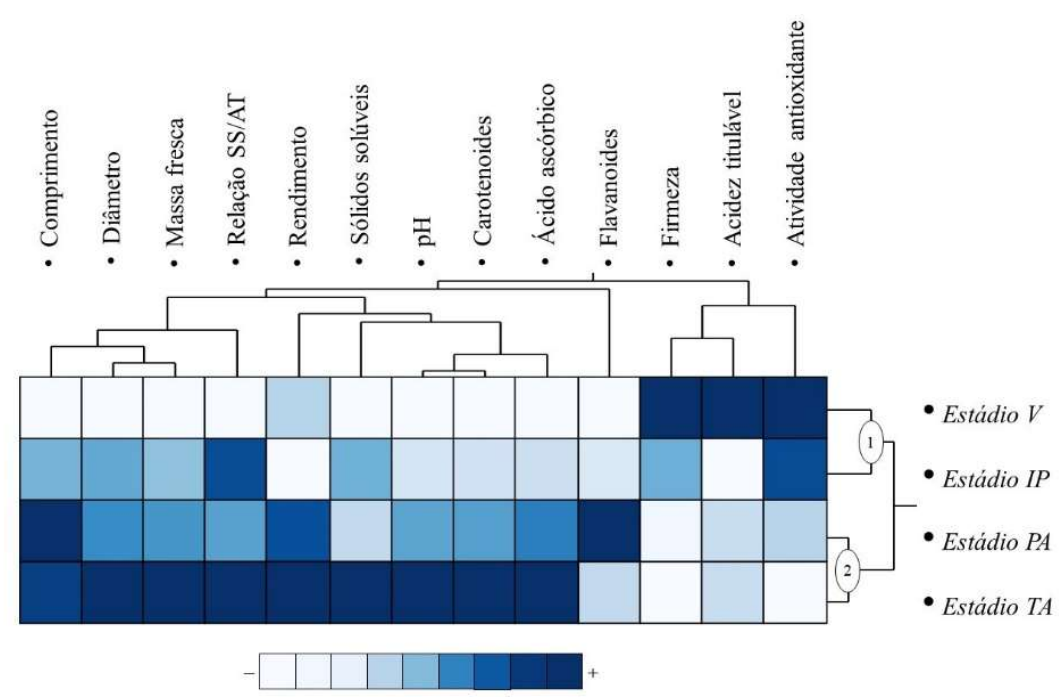

Figura 2. Mapa de cores de agrupamento para diferentes estádios de maturação a partir de atributos físicos, físico-químicos, compostos bioativos e capacidade antioxidante de frutos da uvaieira (Eugenia pyriformis Cambess).

\section{Discussão \\ Atributos físicos}

A determinação de parâmetros físicos de qualidade em frutas nativas e não tradicionais se faz necessário para fornecer dados importantes a respeito da potencialidade destes para o consumo fresco ou para a industrialização, quando associados a caracteres físico-químicos.

O comprimento e diâmetro médio dos frutos de uvaieira nos estádios de maturação avaliados neste trabalho foram superiores aos reportados por Silva et al. (2018b), cujas dimensões foram de 22,0 $\mathrm{mm}$ de comprimento e $27,5 \mathrm{~mm}$ de diâmetro, para frutos de uvaieira colhidos maduros. A massa fresca média dos frutos de uvaieira avaliados neste trabalho foi superior ao reportado por Silva et al. (2018a), cujo valor médio foi de $8,25 \mathrm{~g}$ para frutos de uvaieira colhidos maduros provenientes do município de Salesópolis, SP.

A firmeza da polpa de um fruto é base para a avaliação da textura, que é tida como atributo de qualidade diretamente relacionado com as alterações nas características físico-químicas, responsáveis pelo sabor e aroma dos frutos (Chitarra \& Chitarra, 2005). A firmeza reduziu com o avanço da maturação dos frutos de uvaieira avaliados neste trabalho. Segundo Kluge et al. (2002), ao longo da maturação, as substâncias pécticas da parede celular perdem sua integridade tornando-se solúveis pela atuação de enzimas hidrolíticas como a pectinametilesterase (PME), resultando no decréscimo da firmeza e, consequente, amaciamento da polpa, o que está associado ao processo de amadurecimento, reduzindo sua vida útil pós-colheita (Khademi et al., 2013).

De acordo com Gondim et al. (2013), o rendimento do fruto está relacionado com a percentagem de polpa, portanto, diretamente proporcionais. O rendimento médio da uvaia neste trabalho foi inferior ao citado por Silva et al. (2018a), com 94,47 e 87,45 \% em frutos de uvaieira grandes e pequenos, respectivamente, provenientes de populações de uvaia cultivadas em Salesópolis, SP. No entanto, os valores de rendimento de polpa obtidos neste estudo foram superiores ao valor mínimo estipulado pelo Padrão de Identidade e Qualidade (PIQ) do Ministério da Agricultura, Pecuária e do Abastecimento (MAPA), que estabelece para industrialização um percentual médio de rendimento de polpa acima de $40 \%$ (Brasil, 1999). 


\section{Atributos físico-químicos}

Os frutos sofrem uma série de modificações durante a maturação não somente nas características físicas, mas também físico-químicas. Nesta fase, ocorre um conjunto de modificações irreversíveis, dentre elas alterações bioquímicas, fisiológicas e sensoriais que culminam com a obtenção de fruto com atributos de qualidade desejáveis. Dentre as modificações fisiológicas, tem-se as modificações na taxa respiratoria, degradação da clorofila em paralelo com a biossíntese de carotenoides, antocianinas e os outros componentes responsáveis pela cor, sabor e aroma (Chitarra \& Chitarra, 2005; Dantas et al., 2016).

Valores semelhantes aos deste estudo para pH de uvaias foram reportados por Silva et al. (2018a), com $\mathrm{pH}$ de 3,06 em frutos grandes de uvaia cultivada em Salesópolis, SP. Resultados próximos de $\mathrm{pH}$ também foram reportados por Sganzerla et al. (2018), que constataram pH médio de 3,45, em polpa de frutos de uvaieira, colhidos maduros no município de Urupema, SC.

Valores médios de pH são apreciadas pela indústria, visto que favorecem a conservação, reduzindo a necessidade da adição de ácido cítrico na formulação, durante o processamento da polpa, de modo a evitar o crescimento de microrganismos, além disso o pH também afeta a cor do produto, uma vez que este tem efeito sobre a expressão da antocianina (Lima et al., 2002; Veberic et al., 2014).

Os valores de acidez titulável dos frutos de uvaieira avaliados neste trabalho, corroboraram com Pereira et al. (2012), que reportaram valores de 2,19 \% ácido cítrico, para este mesmo fruto quando maduro. Valores inferiores foram citados por Zillo et al., (2013), Zillo et al., (2014) e Silva et al. (2018a), Silva et al. (2018b) e Sganzerla et al. (2018), que reportaram para frutos maduros de uvaieira valores médios de acidez titulável de $1,17 \%, 1,05 \%, 1,25 \%, 1,67 \%, 1,29 \%$ e 0,75 \% de ácido cítrico, respectivamente.

De acordo com Moura et al. (2011), a acidez é um parâmetro importante para a apreciação do estado de conservação de produtos alimentícios. Visto que no processo de decomposição, seja por hidrólise, oxidação ou fermentação, normalmente, ocorre a alterações na concentração de ácidos orgânicos, por consequência, alterando a acidez do produto.

Os teores de sólidos solúveis dos frutos de uvaieira encontrados neste trabalho, foram superiores aos observados por Zillo et al. (2013), Zillo et al. (2014) e Sganzerla et al. (2018), que constataram valores médios de $6,26 \%, 7,03 \%$, e 4,60 \%, respectivamente. Valores próximos foram citados por Pereira et al. (2012) e Silva et al. (2018a), com médias de 7,70 \% e 7,75 \%, respectivamente, para frutos de uvaieira colhidos maduros.

O teor de sólidos solúveis está relacionado aos compostos orgânicos dissolvidos no suco celular como carboidratos, vitaminas e principalmente açúcares. Durante o processo de maturação dos frutos, os teores tendem a aumentar, tornando-os mais adocicados, devido à solubilização do amido em açúcares menores como glicose e frutose ou mesmo pela perda de água que favorece o acúmulo de açúcares na polpa (Chitarra \& Chitarra, 2005; Anches et al., 2018).

A relação sólidos solúveis/acidez titulável dos frutos de uvaieira foi inferior ao reportado por Pereira et al. (2012), Silva et al. (2018b) e Sganzerla et al. (2018) que descreveram médias de 6,58, 6,14 e 6,13, respectivamente, para frutos de uvaia maduros. A relação SS/AT é tida como um parâmetro para avaliar a qualidade dos frutos, sendo um indicativo do sabor por representar de modo mais significativo, o equilíbrio entre esses dois componentes devendo-se especificar o teor mínimo de sólidos solúveis e o máximo de acidez (Chitarra \& Chitarra, 2005).

\section{Compostos bioativos e atividade antioxidante}

A determinação de compostos como o ácido ascórbico, carotenoides totais, flavonoides amarelos e capacidade antioxidante em frutos nativos necessita de mais aprofundamente. Assim, faz-se necessário gerar informações acerca do potencial funcional de modo a agregar valor a estes frutos. Segundo Sacramento e Souza (2000), as frutas são fontes de calorias, sais minerais, vitaminas, fibras e água, de compostos biativos, o que reforça a importância de conhecer as características físico-químicas da polpa de determinado fruto, visto que o conteúdo presente varia de acordo com a espécie, e também depende de fatores genéticos, local de ocorrência, época de colheita, estádio de maturação, tratos culturais, entre outros.

O conteúdo de ácido ascórbico dos frutos de uvaieira encontrados neste trabalho foi semelhante ao reportado por Stieven et al. (2007), que obtiveram valores médios de variando de 1,69 a 3,99 mg $100 \mathrm{~g}^{-1}$ de ácido ascórbico em polpa congelada. Porém, apresentaram-se inferiores aos reportados por Quináia e Ferreira 
(2007) e Rufino et al. (2009), com média de 38,0 e 39,3 mg $100 \mathrm{~g}^{-1}$ e de ácido ascórbico, respectivamente, para frutos de uvaieira.

Os resultados de carotenoides totais encontrados para os frutos de uvaieira neste trabalho foram superiores aos citados por Rufino et al. (2010) e Zillo et al (2014), que reportaram valores médios de carotenoides totais de $1,70 \mathrm{mg} 100 \mathrm{~g} \mathrm{~g}^{-1}$, e $0,910 \mathrm{\mu g} \mathrm{g}^{-1}$, respectivamente, para frutos de uvaieira. No entanto, inferiores aos citados por Pereira et al. (2012) e Silva et al. (2014) que descreveram níveis elevados variando entre $909,33 \mathrm{\mu g} \mathrm{g}^{-1}$ e $1306,6 \mu \mathrm{g} 100 \mathrm{~g}^{-1}$ de massa fresca, respectivamente.

De acordo com Rodriguez-Amaya (2001), o conteúdo de carotenoides dos vegetais pode ser afetado por diversos fatores, sejam eles inerentes aos vegetais (grau de maturação de um fruto, parte da planta consumida, condições de processamento e armazenamento), métodos de manejo empregados na cultura (condições de cultivo, variedade dos vegetais e uso de agrotóxicos), como também a condições edafoclimáticas (exposição à luz solar, tipo de solo e condições climáticas).

Os compostos fenólicos agem no mecanismo de defesa dos vegetais, sendo os fenóis responsáveis pela ação protetora e antioxidante, dentre eles os flavonoides, que são produzidos na rota do ácido chiquímico, sendo encontrado nos frutos, vegetais, sementes, flores e cascas (Soares, 2002; Yao et al., 2004). Atualmente, é motivo de estudos, devido sua ação antioxidante, o que traz benéficos a saúde humana.

O conteúdo de flavonoides amarelos de uvaia encontrados neste trabalho foi semelhante ao reportado

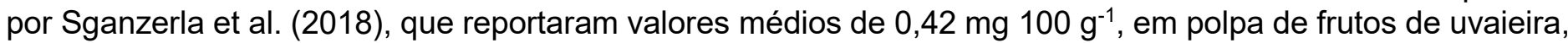
colhidos maduros no município de Urupema, SC. Porém, foi inferior ao citado por Rufino et al. (2010), que

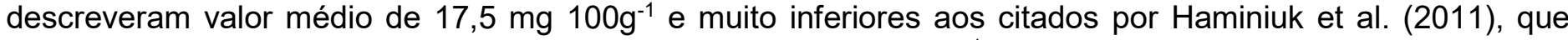

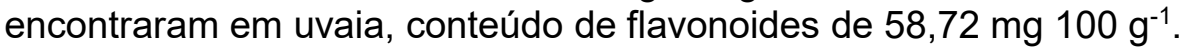

A variação dos flavonoides amarelos juntamente com os carotenoides é um dos indicadores do grau de maturação em que um fruto se encontra. Esses pigmentos são responsáveis pela coloração amarela e alaranjada tanto da casca como da polpa de um fruto (Chitarra \& Chitarra, 2005). De acordo com Zillo et al. (2014) os carotenoides são os pigmentos responsáveis pela coloração característica da uvaia, além disso, desempenha um importante papel na manutenção da saúde, sendo de grande interesse para indústria de alimentos, podendo ser utilizado como corante natural.

O aumento da atividade antioxidante total (AAT) durante a maturação dos frutos de uvaieira neste trabalho, também foi observado por Wang et al. (2009), que ao avaliaram a influência da luz e da maturidade na qualidade de framboesas vermelhas, concluíram que AAT foi superior em frutos totalmente maduros.

Constatou-se que a AAT de uvaia aumentou com o avanço da maturação, notando-se que seu aumento se deu juntamente com o aumento nos conteúdos de carotenoides e flavonoides.

Resultados próximos foram reportados por Rufino et al. (2010), que descreveram um valor médio de 3247 de polpa $\mathrm{g}^{-1}$ de DPPH, em frutos de uvaieira maduros. Mas, considerando que maiores valores de ATT correspondem a maior consumo de polpa, portanto menor atividade antioxidante, os valores foram inferiores aos citados por Pereira et al. (2012) para este mesmo fruto $\left(170,26 \mathrm{~g}\right.$ de polpa $\mathrm{g}^{-1}$ de DPPH). Haminiuk et al. (2011), avaliando a capacidade antioxidante de extratos etanólicos de sete frutas da Mata Atlântica brasileira, constataram que a jabuticaba (Myrciaria cauliflora) e uvaia apresentaram as maiores capacidades antioxidantes medidas pelos métodos de DPPH e b-caroteno-linoléico.

\section{Análise multivariada}

De acordo com Rodrigues et al. (2010), as técnicas de análise multivariada permitem avaliar a variância entre genótipos e selecionar as características mais importantes, na discriminação, por exemplo de acesso de um banco de germoplasma. Dentre as técnicas estatísticas multivariadas, destacam-se os métodos de agrupamento e as correlações entre diferentes variáveis (Silva et al., 2012).

A análise multivariada com base no agrupamento hierárquico mostrou que a maturação da uvaia está dividida em suas fases, a primeira compreende os estádios I e II e a segunda os estádios III e IV. A modificações da primeira fase foram decorrentes das mudanças nos atributos físicos e físico-químicos, enquanto a segunda fase as alterações marcantes foram nos compostos bioativos e atividade antioxidante. Neste sentido, observouse correlação negativa forte entre o conteúdo de carotenoides e flavonoides da polpa e a capacidade antioxidante total da polpa de uvaia, indicando que atividade antioxidante aumenta com o acúmulo destes compostos. Gondim (2012) e Silva et al. (2012), também reportaram essa correlação entre os conteúdos de carotenoides e polifenóis extraíveis totais, com a capacidade antioxidante total durante a maturação de frutos 
de umbuzeiro (Spondias tuberosa Arr. Cam.) e cajazeira (Spondias mombin L.), respectivamente. Dudonné et al. (2009), afirmaram que a presença de compostos fenólicos em extratos de vegetais apresenta alta correlação positiva com a capacidade antioxidante.

\section{Conclusões}

Os frutos da uvaieira apresentam aumento no diâmetro e comprimento e na massa fresca, com o avanço da maturação.

A uvaia é um fruto cuja acidez diminui com a maturação, paralelamente ao aumento nos sólidos solúveis.

A capacidade antioxidante total aumenta com o avanço da maturação da uvaia, mediante o aumento dos teores de carotenoides e flavonoides, que determina as principais mudanças nos estádios finais da maturação deste fruto.

\section{Agradecimentos}

Ao Conselho Nacional de Desenvolvimento Científico e Tecnológico (CNPq) pelo auxílio financeiro na execução do presente trabalho e pela concessão da bolsa de iniciação cientifica ao primeiro autor.

\section{Referências}

ALI, S.S.; KASOJU, N.; LUTHRA, A.; SINGH, S.; SHARANABASAVA, H.; SAHU, A.; BORA U. Indian medicinal herbs as sources of antioxidants. Food Research International, v.41, n.1, p.1-15, 2008. https://doi.org/10.1016/j.foodres.2007.10.001

ANDERSEN, O.; ANDERSEN, V.U. As frutas silvestres brasileiras. 3. ed. São Paulo: Globo, 79 1989. 203 p. AOAC. Official methods of analysis of the Association of Official Analytical Chemistry. $20^{\text {th }}$, Washington: AOAC, 2016.

BRASIL. Ministério da Agricultura, Pecuária e do Abastecimento. Instrução Normativa ${ }^{\circ} 122$, de 10 de setembro de 1999. Diário Oficial da República Federativa do Brasil, Brasília, 13 de setembro de 1999. Seção 1. Brasília: MAPA, 1999. p.72-76.

BRASIL. Normas Analíticas do Instituto Adolfo Lutz: Métodos químicos e físicos para análise de alimentos. São Paulo: IAL, 2008. 919p.

CHITARRA, M.I.F.; CHITARRA, A.D. Pós-colheita de frutas e hortaliças: fisiologia e manuseio. Lavras: UFLA, 2. ed., 293p. 2005.

DANTAS, A.L.; SILVA, S.M.; DANTAS, R.L.; SOUSA, A.S.B.; SCHUNEMANN, A.P.P. Desenvolvimento, fisiologia da maturação e indicadores do ponto de colheita de frutos da umbugueleira (Spondias sp.). Revista Brasileira de Fruticultura, v. 38, n. 1, p. 033-042, 2016. http://dx.doi.org/10.1590/0100-2945-271/14

DUDONNÉ, S.; VITRAC, X.; COUTIERE, P.; WOILLEZ, M.; MÉRILON, J.M. Comparative Study of Antioxidant Properties and Total Phenolic Content of 30 Plant Extracts of Industrial Interest Using DPPH, ABTS, FRAP, SOD, and ORAC Assays. Journal of Agricultural and Food Chemistry, v.57, n.5, p.1768-1774, 2009. http://dx.doi.org/10.1021/jf803011r

EGEA, M.B. Frutos nativos da Floresta Atlântica com potencial de maior utilização pela população e pela indústria: caracterização nutracêutica e aromática. 2014. Tese (Doutorado em Engenharia de Alimentos). Universidade Federal do Paraná, Curitiba, 2014.

FERREIRA, D.F. SISVAR: A computerstatisticalanalysis system. Ciência \&Agrotecnologia, v.35, n.6, p.10391042, 2011.http://dx.doi.org/10.1590/S1413-70542011000600001

FRANCIS, F.J. Analysis of anthocyanins. In: MARKAKIS, P. (ed.). Anthocyanins as food colors. New York: Academic Press, p.181-207, 1982. 
GONDIM, P.J.S. Identificação de carotenoides e quantificação de compostos bioativos e atividade antioxidante em frutos do gênero Spondias. 2012. 104f. Tese (Doutorado em Agronomia) - Universidade Federal da Paraíba. Areia, 2012.

GONDIM, P.J.S.; SILVA, S.M.; PEREIRA, W.E.; DANTAS, A.L.; CHAVES NETO, J.R.; SANTOS, L.F. Qualidade de frutos de acessos de umbu-cajazeira (Spondias sp.). Revista Brasileira de Engenharia Agrícola e Ambiental, v.17, n.11, p.1217-1221, 2013. http://dx.doi.org/10.1590/S1415-43662013001100013

HAMINIUK, C.W.I.; PLATA-OVIEDO, M.S.V.; GUEDES, A.R.; STAFUSSA, A.P.; BONA, E.S.T. Chemical, antioxidant and antibacterial study of Brazilian fruits. International Journal of Food Science \& Technology, v.46, n.7, p.1529-1537, 2011. http://dx.doi.org/10.1111/j.1365-2621.2011.02653.x

HIGBY, W.K. A simplified method for determination of some the carotenoid distribuition in natural 85 and carotene-fortified orange juice. Journal of Food Science, v.27, n.1, p.42-49, 1962. https://doi.org/10.1111/j.1365-2621.1962.tb00055.x

JACOMINO, A.P.; SILVA, A.P.G.; FREITAS, T.P.; MORAIS, V.S.P. Uvaia - Eugenia pyriformis Cambess. In: RODRIGUES, S.; SILVA, E.O.; DE BRITO, E.S. Exotic Fruits Reference Guide. 1. ed. Londres: Elsevier, 2018. p. 435-438.

KHADEMI, O.Z.; ZAMANI, E.P.A.; KALANTARI, E.S. Effect of UV-C radiation on postharvest physiology of persimmon fruit (Diospyros kaki Thunb.) cv. 'Karaj' during storage at cold temperature. International Food Research Journal, v.20, n.1, p.247-253, 2013.

KLUGE, R.A.; NACHTIGAL, J.C.; FACHINELLO, J.C.; BILHALVA, A.B. Fisiologia e manejo pós-colheita de frutas de clima temperado. 2. ed. Pelotas: UFPEL, 2002. 163p.

LANDRUM, L.R.; KAWASAKI, M.L. The genera of Myrtaceae in Brazil: an Illustrated synoptic treatment and identification keys. Brittonia, v.49, n.4, p.508, 1997. http://dx.doi.org/10.2307/2807742

LIMA, E.D.P.A.; LIMA, C.A.A.; ALDRIGUE, M.L.; GONDIM, P.J.S. Umbu-cajá (Spondiasspp) aspectos de pós-colheita e processamento. João Pessoa: Ed Universitária/ldeia, 2002. 57p.

LIMA, E.D.P.A.; LIMA, C.A.A.; ALDRIGUE, M.L.; GONDIM, P.J.S. Caracterização física e química dos frutos da umbu-cajazeira (Spondiasspp.) em cinco estádios de maturação, da polpa congelada e néctar. Revista Brasileira de Fruticultura, v.24, p.338-343. 2002.http://dx.doi.org/10.1590/S0100-29452002000200013

LORENZI, H. Árvores Brasileiras: Manual de identificação e cultivo de plantas arbóreas nativas do Brasil, Instituto Plantarum, Nova Odessa. 2000.

LU, M., YUAN, B., ZENG, M., CHEN, J. Antioxidant capacity and major phenolic compounds of spices commonly consumed in China. Food Research International, v.44, n.2, p.530-536. 2011. http://dx.doi.org/10.1016/j.foodres.2010.10.055

MATTOS, J. R. Uvalheira: fruteiras nativas do Brasil. Porto Alegre: Instituto de Pesquisa de 89 Recursos Naturais e Renováveis, 1988, 36 p.

MOURA, C.L.A.; PINTO, A.S. FIGUEIREDO, R.W. Processamento e utilização da polpa de cajá (Spondias mombin L.). B. CEPPA, v.29, n.2, p.237-252, 2011. http://dx.doi.org/10.5380/cep.v29i2.25489

PEREIRA, M.C.; STEFFENS, R.S.; JABLONSKI, A.; HERTZ, P.F.; RIOS, A.O.; VIZZOTTO, M.; FLORES, S.H. Characterization and Antioxidant Potential of Brazilian Fruits from the Myrtaceae Family. Journal of Agricultural and Food Chemistry, v.60, n.12, p.3061-3067, 2012. http://dx.doi.org/10.1021/jf205263f

QUINÁIA, S.P.; FERREIRA, M. Determination of ascorbic acid in pharmaceutical formulations and tropical juices by means of spectrophotometric titration. Revista Ciências Exatas Naturais, v.9, n.1, p.41-50, 2007.

RODRIGUES, H.C.A.; CARVALHO, S. P.; CARVALHO, A.A.; FILHO, J.L.S.C.; CUSTÓDIO, T.N. Avaliação da diversidade genética entre acessos de mamoneira (RicinuscommunisL.) por meio de caracteres morfoagronômicos. Revista Ceres, v.57, n.6, p.773-777, 2010. http://dx.doi.org/10.1590/S0034-

$37 \times 2010000600012$. 
RODRIGUEZ-AMAYA, D.B. A guide to carotenoid analysis in food. International Life Sciences Institute, Washington, D.C. 64p., 2001.

RUFINO, M.S.M.; ALVES, R.E.; BRITO, E.S.; PÉREZ-JIMÉNEZ, J.; SAURA-CALIXTO, F.; MANCINI-FILHO, J. Bioactive compounds and antioxidant capacities of 18 non-traditional tropical fruits from Brazil. Food Chemistry, v.121, n.4, p.996-1002, 2010. http://dx.doi.org/10.1016/j.foodchem.2010.01.037

RUFINO, M.S.M.; FERNANDES, F.A.N.; ALVES, R.E.; BRITO, E.S. Free redical-scavenging behaviour of some North-east Brasilian fruits in a DPPH system. Food Chemistry, v.114, n.2, p.693-695, 2009. http://dx.doi.org/10.1016/j.foodchem.2008.09.098

SACRAMENTO, C.K.; SOUZA, F.X. Cajá (Spondias mombin L.). (Série Frutas Nativas, 4). Jabuticabal: Funep, 2000. 42 p.

SANCHES, A.G.; SILVA, M.B.; MOREIRA, E.G.S.; SANTOS, E.X. Atraso na maturação e qualidade póscolheita de seriguela exposta a radiação ultravioleta-C. Nativa, v.6, n.3, p.225-232, 2018. http://dx.doi.org/10.31413/nativa.v6i3.4498

SCALON, S.P.Q.; DELL'OLIO, P.; FORNASIERI, J.L. Temperatura e embalagens na conservação pós-colheita de Eugenia uvalha Cambess - Mirtaceae. Ciência Rural, v.34, n.6, p.1965-1968, 2004. http://dx.doi.org/10.1590/S0103-84782004000600048

SELLAPPAN, S.; AKOH, C.C.; KREWER, G. Phenolic compounds and antioxidant capacity of Georgia-Grown blueberries and blackberries. Journal of Agricultural and Food Chemistry, v.50, p.2432-2438, 2002. http://dx.doi.org/10.1021/jf011097r

SGANZERLA, W.G.; BELING, P.C.; FERRAREZE, J.P.; KOMATSU, R.A.; NUNES, M.R.; VEECK, A.P.L. Nutritional, physicochemical and antimicrobial properties of uvaia pulp (Eugenia pyriformis Cambess). Communications in Plant Sciences, v.8, n.1, p.1-7, 2018. http://dx.doi.org/10.26814/cps2018001

SILVA, A.P.G.; ESPINDOLA, I.C.; MIGUEL, A.C.A.; SPRICIGO, P.C.; SARTORI, S.; JACOMINO, A.P. Postharvest storage of two accessions of uvaia (Eugenia pyriformis Cambess) at room temperature. Acta Horticulturea, n.1194, p. 959-964, 2018a. http://dx.doi.org /10.17660/ActaHortic.2018.1194.136

SILVA, A.P.G.; TOKAIRIN, T.O.; ALENCAR, S.M.; JACOMINO, A.P. Characteristics of the fruits of two uvaia populations grown in Salesópolis, SP, Brazil. Revista Brasileira de Fruticultira, v.40, n.2, p.1-7, 2018b. http://dx.doi.org /10.1590/0100-29452018511

SILVA, D.F.P.; SIQUEIRA, D.L.; ROCHA, A.; SALOMÃO, L.C.C.; MATIAS, R.G.P.; STRUIVING, T.B. Diversidade genética entre cultivares de mangueiras, baseada em caracteres de qualidade dos frutos. Revista Ceres, v.59, n.3, 225-232, 2012. http://dx.doi.org/10.1590/S0034-737X2012000200011

SILVA, F.V.G.; SILVA, S.M.; SILVA, G.C.; MENDONÇA, R.M.N.; ALVES, R.E.; DANTAS, A.L. Bioactive compounds and antioxidant activity in fruits of clone and ungrafted genotypes of yellow mombin tree. Ciência Tecnologia de Alimentos, v.32, n.4, p.685-691, 2012. http://dx.doi.org/10.1590/S0101-20612012005000101

SILVA, N.A.; RODRIGUES, E.; MERCADANTE, A.Z.; ROSSO, V.V. Phenolic compounds and carotenoids from four fruits native from the Brazilian Atlantic forest. Journal of Agricultural and Food Chemistry, v.62, n.22, p.5072-5084, 2014. http://dx.doi.org/10.17660/ActaHortic.2018.1194.136

SOARES, S.E. Ácidos fenólicos como antioxidantes. Revista Nutrição de Campinas, v. 1, n. 15, p. 71-81, 2002. http://dx.doi.org/10.1590/S1415-52732002000100008

STROHECKER, R.; HENNING, H.M. Analisis de vitaminas: métodos comprobados. Madrid: Paz Montalvo, 1967. 428p.

VEBERIC, R.; STAMPAR, F.; SCHMITZER, V.; CUNJA, V.; ZUPAN, A.; KORON, D.; MIKULIC-PETKOVSEK, $M$. Changes in the contents of anthocyanins and other compounds in blackberry fruits due to freezing and longterm frozen storage. Journal of Agricultural and Food Chemistry, v.62, n.29, p.6926-6935, 2014. http://dx.doi.org/10.1021/jf405143w.PMid:24422506 
WANG, S.Y.; CHEN, C.; WANG, C.Y. The influence of light and maturity on fruit quality and flavonoid content of red raspberries. Food Chemistry, v.112, n.3, p.676-684, 2009. http://dx.doi.org/10.1016/j.foodchem.2008.06.032

YAO, L.H.; JIANG, Y.M.; SHI, J.; TOMÁS-BARBERÁN, F.A.; DATTA, N.; SINGANUSONG, R.; CHEN, S.S. Flavonoids in food and their health benefits. Plant Foods for Human Nutrition, v.59, n.3, p.113-122, 2004. http://dx.doi.org/10.1007/s11130-004-0049-7

ZILLO, R.R.; SILVA, P.P.M.; ZANATTA, S.; CARMO, L.F.; SPOTO, M.H.F. Parâmetros físico-químicos e sensoriais de polpa de uvaia (Eugenia Pyriformis) submetidas à pasteurização. Bioenergia em revista: diálogos, v.4, n.2, p.20-33, 2014.

ZILLO, R.R.; SILVA, P.P.M.; ZANATTA, S.; CARMO, L.F.; SPOTO, M.H.F. Qualidade físico-química da fruta in natura e da polpa de uvaia congelada. Revista Brasileira de Produtos Agroindustriais, v.15, n.3, p.293-298, 2013. http://dx.doi.org/10.15871/15178595/rbpa.v15n3p293-298. 